\title{
Tumor adrenocortical metastático, uma apresentação atípica: relato de caso
}

Rodrigo Firmino Schirmbeck Moraes 1, Julia Pastorello 2, Emanuela Lando 3, Camila dos Santos do Amaral 2, Cristiane Pagnussat Cechetti 2, Marina Ractz Bueno 2, Giovana Zerwes Vacaro 2, Nicoli Taiana Henn 2, Douglas Carvalho Cogo 2, Robson Rottenfusser 4, Alexandre Bueno da Silva 5, Eduardo Lima Tigre 5

${ }^{1}$ Médico Residente em Cirurgia Geral, Hospital de Clínicas de Passo Fundo - HCPF, Passo Fundo, Rio Grande do Sul/RS, Brasil.

${ }^{2}$ Departamento de Oncologia Clínica, Hospital de Clínicas de Passo Fundo - HCPF, Passo Fundo, Rio Grande do Sul/RS, Brasil.

${ }^{3}$ Acadêmica de Medicina, Faculdade Meridional - IMED, Passo Fundo, Rio Grande do Sul/RS, Brasil.

${ }^{4}$ Médico Radiologista, Hospital de Clínicas de Passo Fundo - HCPF, Passo Fundo, Rio Grande do Sul/RS, Brasil.

${ }^{5}$ Departamento de Cirurgia Vascular, Hospital de Clínicas de Passo Fundo - HCPF, Passo Fundo, Rio Grande do Sul/RS, Brasil.

*Autor Correspondente: Emanuela Lando. Rua Paisandú,358. Cep: 99010100 - Passo Fundo, Rio Grande do Sul/RS. Brasil. Fone: +55 (54) 9 91861467. E-mail: manu.lando@hotmail.com.

Research Ethics Committee Approval (if necessary): Declaramos que o paciente aceitou a divulgação de informação presente no estudo através de termo de consentimento informado e que o estudo seguiu as diretrizes éticas estabelecidas pela Declaração de Helsinque.

Received on: Sep 9, 2021. Accepted on: Sep 22, 2021. Available online: Nov 3, 2021.

\section{Resumo}

O carcinoma adrenal corresponde a uma neoplasia rara representando cerca de $0,05 \%$ a $0,2 \%$ do total de malignidades já descritas, sendo que sua atividade metastática se apresenta em cerca de $30 \%$ a 50\% dos casos, com maior incidência para disseminação hepática e linfonodos retroperitoniais. O presente trabalho visa relatar um caso raro relacionado a associação de tumor adrenocortical metastático com a apresentação inicial de trombose, relação essa pouco descrita em literatura médica vigente, tornando-se, assim, um desafio na prática da oncologia moderna.

Palavras- chave: Oncologia; Carcinoma Adrenal; Tromboembolismo; Neoplasia.

\section{Introdução}

O carcinoma adrenal é considerado uma neoplasia rara, correspondendo a cerca de $0,05 \%$ a $0,2 \%$ do total de malignidades já descritas, apresentando associação com síndromes genéticas tais como Li- Fraumeni, Beckwith-Weideman e neoplasia endócrina múltipla do tipo I [1-4]. A atividade metastática do carcinoma adrenal apresenta-se em cerca de $30 \%$ a 
$50 \%$ dos casos, com maior incidência para disseminação hepática e linfonodos retroperitoniais em relação aos ademais sítios [1-2].

O tratamento para os casos de carcinoma adrenal depende do estadiamento e do performance status do paciente, sendo que a ressecção cirúrgica é indicada como tratamento de primeira escolha quando a doença encontra-se localizada e o tratamento de eleição quando casos disseminados são evidenciados $\mathrm{o}$ fármaco mitotano $\mathrm{e}$ quimioterapia baseada em cisplatina, etoposide, 5-fluoracila, estreptozotocina e doxorrubicina tornam se o tratamento de escolha [2-4].

Sendo assim, o presente trabalho visa relatar um caso raro da associação diagnóstica da neoplasia apresentada, carcinoma adrenocortical com o quadro de trombose inicial, relação essa pouco descrita em literatura médica vigente. Assim tornando o presente caso um desafio na prática da oncologia moderna.

\section{Relato de Caso}

Paciente, sexo feminino, 29 anos, compareceu ao departamento de emergência hospitalar devido quadro de edema bilateral em membros inferiores acentuado à esquerda, associada a quadro álgico em região inguinal ipsilateral, sendo diagnosticada com trombose venosa profunda em membro inferior esquerdo, iniciando, assim, tratamento preconizado em anticaogulação.

Ao exame de angiotomografia abdominal, foi evidenciado umapresença de massa adrenal direita de 20,6 x13,0 x26,3 cm relacionada à lesão neoplásica primária, estendendose para veia cava inferior e veias renais, obliterando a luz vascular (Figura 1).

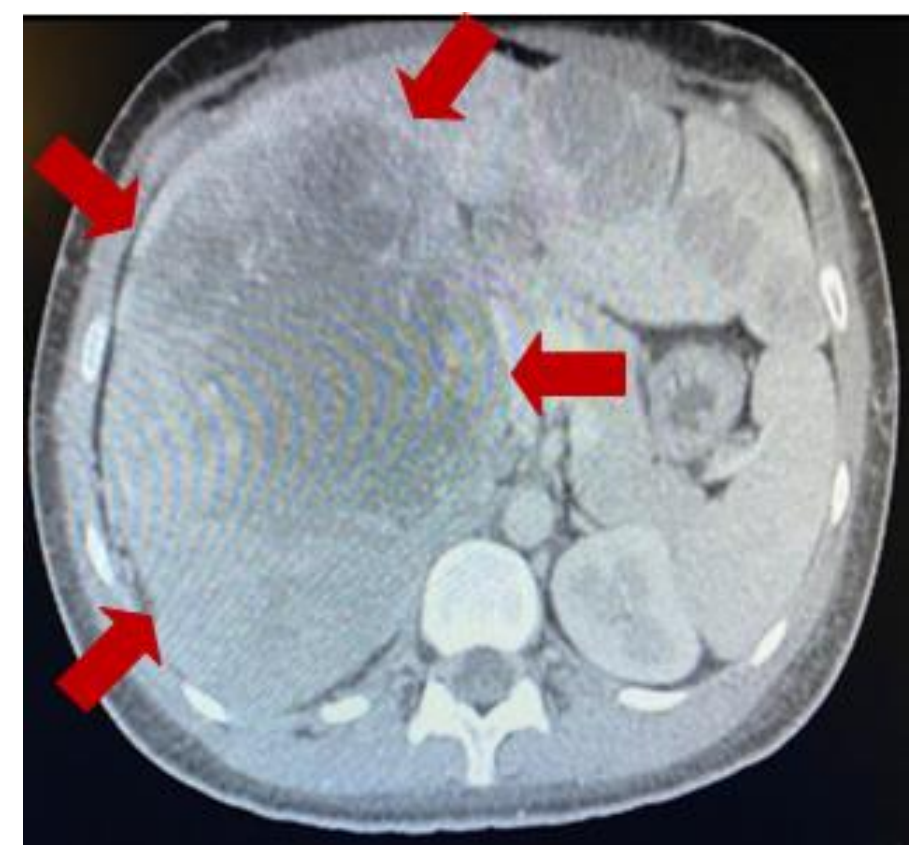

Figura 1: Angiotomografia abdominal, evidenciou presença de massa adrenal direita de 20,6 ×13,0 ×26,3 cm (setas vermelhas) relacionada à lesão neoplásica primária. 
Adicionalmente,

foram identificados múltiplos nódulos hepáticos, sendo o maior de 4,6 x 4,4 cm no segmento VII hepático, corroborando com atividade metastática, além da presença de falha de preenchimento da veia cava inferior infra-renal e sua porção a jusante, bem como da ilíaca comum direita e veias ilíacas comuns externas e internas esquerdas por material hipodenso, relacionado a trombo hemático.

A investigação foi continuada sendo realizado exame de angiotomografia de tórax o qual revelou tromboembolismo pulmonar em ramos secundários do lobo inferior da artéria pulmonar esquerda e numerosos nódulos em ambos os pulmões, relacionados a implantes metastáticos, além de conglomerado linfonodal em região hilar direita de 4,1×2,1 cm (Figura 2).

Sendo que o exame de tomografia computadorizada evidenciou a falha de enchimento hipodensa na veia cava inferior e nas veias ilíacas comuns compatível com trombo hemático (Figura 3).

O exame anatomopatológico hepático evidenciou neoplasia de células redondas. Todavia, o exame imunohistoquímico confirmou o diagnóstico de carcinoma adrenocortical direito metastático e positividade para Sinaptofisina, Inibina e Melan A. Devido a presença de doença metastática, seguiu-se tratamento inicialmente baseado em Mitotano, o qual, posteriormente, foi incluído ao esquema quimioterápico preconizado com Cisplatina, Etopiside e Doxorrubicina, o qual a paciente ainda está em vigência.

A paciente foi acompanhada desde o início de diagnóstico neoplásico até o presente momento qual encontrase em tratamento oncológico quimioterápico.

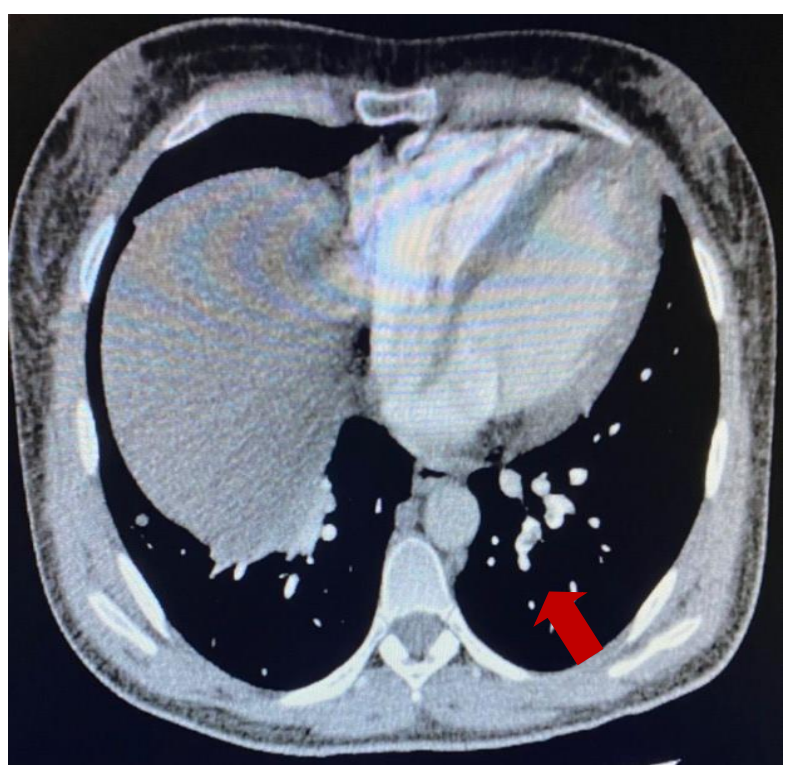

Figura 2: Angiotomografia de tórax, revelou região onde há sinais de tromboembolismo pulmonar em ramos secundários do lobo inferior da artéria pulmonar esquerda (seta vermelha). 


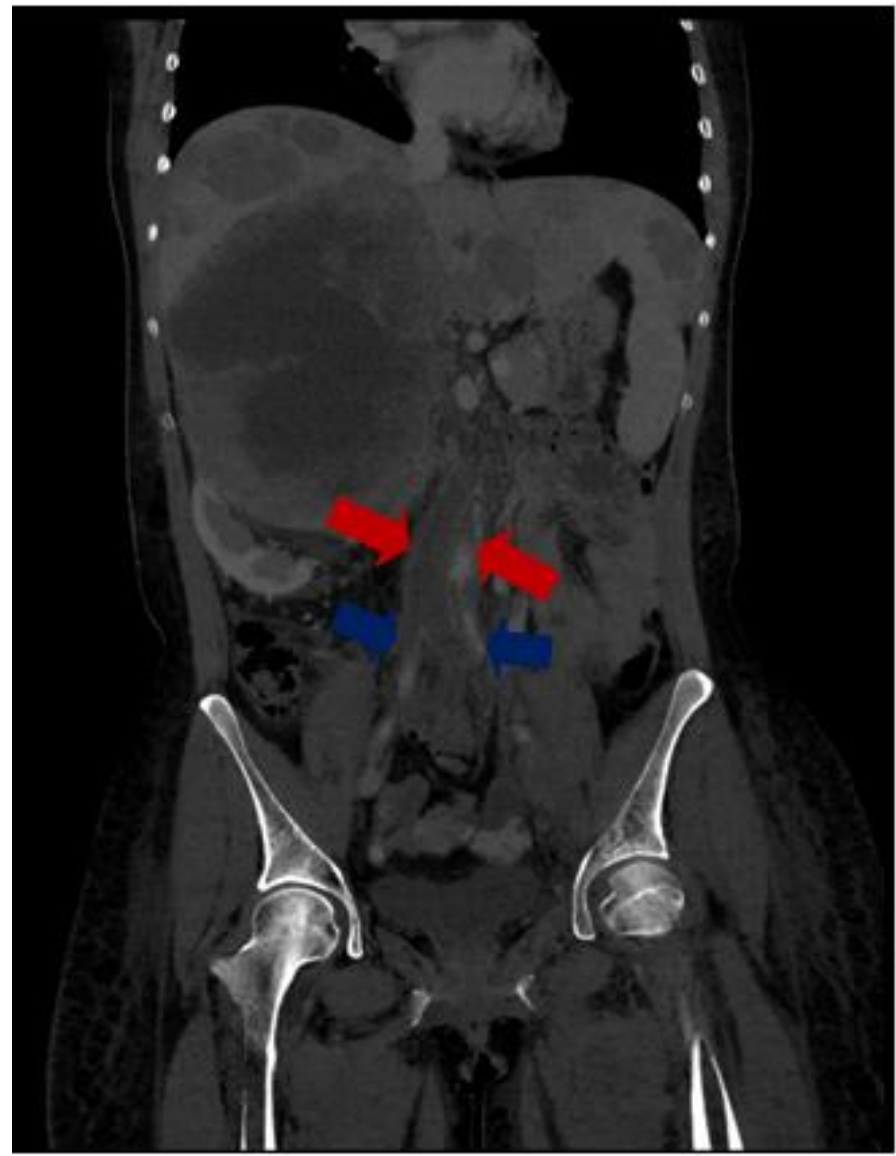

Figura 3: Tomografia computadorizada em reconstrução no plano coronal em intensidade de projeção mínima demonstrando falha de enchimento hipodensa na veia cava inferior (setas vermelhas) e nas veias ilíacas comuns (setas azuis), compatível com trombo hemático. Adicionalmente, observa-se massa na loja adrenal direita.

\section{Discussão e Conclusão}

O carcinoma adrenal é considerado uma neoplasia rara, originado da região do córtex da glândula adrenal, apresentando taxa de incidência preferenciais em relação a idade dos pacientes acometidos. Destaca-se que o carcinoma adrenal se manifesta na população infantil, especialmente entre o primeiro e segundo ano de vida, na população infanto- juvenil entre os 7 aos 16 anos e na população adulta geralmente na quinta década de vida [1-3].
Tal neoplasia representa cerca de $0,05 \%$ a $0,2 \%$ do total de malignidades já descritas na literatura médica/ oncológica vigente [2-4]. Clinicamente, os principais sintomas apresentados são evidenciados por quadro álgico abdominal associado a massa tumoral, fadiga, perda de peso, episódios febris e hematúricos. Ademais, tal neoplasia apresenta associação com síndromes genéticas como Li- Fraumeni, BeckwithWeideman e neoplasia endócrina múltipla do tipo I [2-4].

O diagnóstico relacionado ao carcinoma adrenal geralmente ocorre 
tardiamente, quando o paciente desenvolve doença tumoral mensurável entre 6 a $20 \mathrm{~cm}$, o que faz com que em certos casos o tumor desloque o rim ipsilateral até a região de fossa ilíaca [57].

Ademais, levando em consideração o quadro de disseminação associada, as vias de preferência são os gânglios linfáticos, em mais de $65 \%$ de suas apresentações, especialmente os retroperitonias, o sítio pulmonar em cerca de $70 \%$, hepática em $4 \%$ e sítio ósseo em aproximadamente $25 \%$ do total das apresentações, corroborando tanto para disseminação linfática quanto hematogênica associada a atividade metastática [8-10].

Em relação ao estadiamento tumoral leva se em consideração exames de imagem como radiografia torácica, ultrassom abdominal, tomografia computadorizada e cintilografia óssea [3-6, 11]. Todavia, o exame de ressonância magnética e a angiotomografia tornam-se de suma importância para avaliar possíveis acometimentos vasculares, especialmente sinais associados a trombose pelo paciente em estadiamento [3-6, 11].

O tratamento para os casos de carcinoma adrenal depende do estadiamento e performance status do paciente, sendo que a ressecção cirúrgica é indicada quando a doença está localizada, sendo o tratamento de eleição frente esses casos [2-4].

Todavia, quanto aos casos metastáticos, a instituição de Mitotano, além de quimioterapia baseada em Cisplatina, Etoposide, 5-Fluoracila,
Estreptozotocina e Doxorrubicina tornase a opção de eleição frente ao tratamento, similiar ao preconizado no caso apresentado [2-4, 12-13]. Ressaltase que, quando instituído o fármaco Mitotano, vê-se que este auxilia na taxa de resposta terapêutica entre 14 a 35\% do total de casos de carcinoma adrenal [12-13].

O tratamento radioterápico é reservado especialmente quando a presença de metástase óssea é evidenciada, qual torna-se tratamento de escolha, sendo sugerido irradiação entre 50 a 60 Gy em região de leito tumoral, conforme diâmetro e índice mitótico para obtenção de ganho de sobrevida livre de progressão [14].

Em relação ao prognóstico, a sobrevida média em pacientes com idade a inferior a 5 anos gira em torno de $49 \%$, sendo que se realizado tratamento cirúrgico tal dado varia até cerca de $70 \%$ [3]. Na população adulta, quando instituído tratamento cirúrgico, a sobrevida média geral varia em torno de $20 \%$ até $58 \%$ e, quando não há possibilidade de tal intervenção, a sobrevida média geral varia de $10 \%$ até $35 \%$ em 5 anos [3].

Sendo assim, o presente caso, chama atenção pela raridade da associação diagnóstica com a apresentação inicial do quadro de trombose, relação essa pouco descrita em literatura médica vigente, tornando, assim, o caso um desafio na prática da oncologia moderna. 


\section{Referências}

[1] Sociedade Brasileira de Endocrinologia e Metabologia e Colégio Brasileiro de Radiologia. Associação Médica Brasileira e Conselho Federal de Medicina. Projeto Diretrizes. Tumores Adrenocorticais. 20 de junho de 2006.

[2] Mansmann G, Lau J, Balk E, Rothberg M, Miyachi Y, Bornstein SR. The clinically inapparent adrenal mass: update in diagnosis and management. Endocr Rev. 2004 Apr;25(2):309-40. doi: 10.1210/er.2002-0031.

[3] Roman S. Adrenocortical carcinoma. Curr Opin Oncol. 2006 Jan;18(1):36-42. doi: 10.1097/01.cco.0000198976.43992.14.

[4] Dackiw AP, Lee JE, Gagel RF, Evans DB. Adrenal cortical carcinoma. World J Surg. 2001 Jul;25(7):914-26. doi: 10.1007/s00268-001-0030-7.

[5] Allolio B, Fassnacht M. Clinical review: Adrenocortical carcinoma: clinical update. J Clin Endocrinol Metab. 2006 Jun;91(6):2027-37. doi: 10.1210/jc.2005-2639.

[6] Allolio B, Hahner S, Weismann D, Fassnacht M. Management of adrenocortical carcinoma. Clin Endocrinol (Oxf). 2004 Mar;60(3):273-87. doi: 10.1046/j.1365-2265.2003.01881.x..

[7] Icard P, Goudet P, Charpenay C, Andreassian B, Carnaille B, Chapuis $\mathrm{Y}$, Cougard P, Henry JF, Proye C. Adrenocortical carcinomas: surgical trends and results of a 253-patient series from the French Association of Endocrine Surgeons study group. World J Surg. 2001 Jul;25(7):891-7. doi: 10.1007/s00268-001-0047-y.
[8] Hutter AM Jr, Kayhoe DE. Adrenal cortical carcinoma. Results of treatment with o,p'DDD in 138 patients. Am J Med. 1966 Oct;41(4):581-92. doi: 10.1016/0002-9343(66)90220-8.

[9] King DR, Lack EE. Adrenal cortical carcinoma: a clinical and pathologic study of 49 cases. Cancer. 1979 Jul;44(1):239-44. doi: 10.1002/10970142(197907)44:1<239::aidcncr2820440139>3.0.co;2-r.

[10] Luton JP, Cerdas S, Billaud L, Thomas G, Guilhaume B, Bertagna X, Laudat $\mathrm{MH}$, Louvel A, Chapuis $\mathrm{Y}$, Blondeau $\mathrm{P}$, et al. Clinical features of adrenocortical carcinoma, prognostic factors, and the effect of mitotane therapy. N Engl J Med. 1990 Apr 26;322(17):1195-201.

doi: 10.1056/NEJM199004263221705.

[11] Szolar DH, Korobkin M, Reittner P, Berghold A, Bauernhofer T, Trummer $H$, Schoellnast $H$, Preidler KW, Samonigg H. Adrenocortical carcinomas and adrenal pheochromocytomas: mass and enhancement loss evaluation at delayed contrast-enhanced CT. Radiology. 2005 Feb;234(2):479-85. doi: 10.1148/radiol.2342031876.

[12] Khorram-Manesh A, Ahlman $\mathrm{H}$, Jansson S, Wangberg B, Nilsson O, Jakobsson CE, Eliasson B, Lindstedt $S$, Tisell LE. Adrenocortical carcinoma: surgery and mitotane for treatment and steroid profiles for follow-up. World J Surg. 1998;22:60511.

[13] Wooten MD, King DK. Adrenal cortical carcinoma. Epidemiology and treatment with mitotane and a review of the literature. Cancer. 1993 Dec 
1;72(11):3145-55. doi: 10.1002/1097-

0142(19931201)72:11<3145::aid-

cncr2820721105>3.0.co;2-n.

[14] Markoe AM, Serber W, Micaily B, Brady LW. Radiation therapy for adjunctive treatment of adrenal cortical carcinoma. Am J Clin Oncol. 1991 Apr;14(2):170-4. doi: 10.1097/00000421199104000-00015.

Conflito de interesse: Os autores declaram não haver conflitos de interesse.

Agradecimentos: Agradecemos a toda equipe pelo excelente manejo multidisciplinar e especialmente ao Hospital de Clínicas de Passo Fundo na contribuição e fornecimento de dados para realização do presente trabalho.

Financiamento: Não se aplica.

Como citar este artigo: Moraes RFS, Pastorello J, Lando E, Amaral CS, Cechetti CP, Bueno MR, Vacaro GZ Henn NT, Cogo DC, Rottenfusser R, Silva AB, Tigre EL. Tumor Adrenocortical metastático, uma apresentação atípica: relato de caso. Brazilian Journal of Case Reports. 2021Oct-Dec;01(4):98-104. 